\title{
Delta signaling from the germ line controls the proliferation and differentiation of the somatic follicle cells during Drosophila oogenesis
}

\author{
Hernán López-Schier and Daniel St. Johnston ${ }^{1}$ \\ Wellcome/CRC Institute and Department of Genetics, University of Cambridge, Cambridge CB2 1QR, UK
}

The body axes of Drosophila are established during oogenesis through reciprocal interactions between the germ line cells and the somatic follicle cells that surround them. The Notch pathway is required at two stages in this process: first, for the migration of the follicle cells around the germ line cyst and, later, for the polarization of the anterior-posterior (A-P) axis of the oocyte. Its function in these events, however, has remained controversial. Using clonal analysis, we show that Notch signaling controls cell proliferation and differentiation in the whole follicular epithelium. Notch mutant follicle cells remain in a precursor state and fail to switch from the mitotic cell cycle to the endocycle. Furthermore, removal of Delta from the germ line produces an identical phenotype, showing that Delta signals from the germ cells to control the timing of follicle cell differentiation. This explains the axis formation defects in Notch mutants, which arise because undifferentiated posterior follicle cells cannot signal to polarize the oocyte. Delta also signals from the germ line to Notch in the soma earlier in oogenesis to control the differentiation of the polar and stalk follicle cells. The germ line therefore regulates the development of the follicle cells through two complementary signaling pathways: Gurken signals twice to control spatial patterning, whereas Delta signals twice to exert temporal control.

[Key Words: Germ line; oogenesis; cell cycle]

Received February 14, 2001; revised version accepted April 13, 2001.

The Notch family of receptors play a central role in an evolutionarily conserved signaling pathway that regulates numerous cell fate decisions in many organisms (Artavanis-Tsakonas et al. 1999). Notch encodes a large transmembrane receptor for the ligands Delta (D1), Serrate (Ser), and related orthologs in other species (collectively called DSL), which are also transmembrane proteins with large extracellular domains, and signaling therefore requires direct cell-cell contact. On ligand binding, Notch receptor undergoes a presenilin-dependent proteolytic cleavage to release the intracellular domain from the membrane (De Strooper et al. 1999; Struhl and Greenwald 1999; Ye et al. 1999). This domain can then translocate to the nucleus, where it acts as a transcriptional activator in association with Suppressor of Hairless (Su[H]; Bailey and Posakony 1995; Lecourtois and Schweisguth 1995).

The best characterized function of Notch in Drosophila is in lateral inhibition during the specification of

${ }^{1}$ Corresponding author.

E-MAIL ds139@mole.bio.cam.ac.uk; FAX 44-(0)-1223-334-089.

Article and publication are at www.genesdev.org/cgi/doi/10.1101/ $\operatorname{gad} .200901$. the neuroblasts and sensory organ precursors of the nervous system (Lehmann et al. 1983; Campos-Ortega and Knust 1990). In this process, one cell in a cluster of equipotent precursor cells expresses higher levels of a Notch ligand, such as Delta, and activates the Notch pathway in the neighboring cells to turn on the expression of the E(spl) complex genes (Bailey and Posakony 1995; Lecourtois and Schweisguth 1995). As these transcription factors inhibit proneural gene expression, this prevents the cells from adopting a neural fate (Oellers et al. 1994). Mutants in this pathway therefore result in a neurogenic phenotype in which too many cells adopt a neural fate at the expense of epidermis. Although Notch signaling usually regulates cell fate determination, in a few cases it has been shown to have other effects on other aspects of cell behavior. For example, the Caenorhabditis elegans Notch homolog, GLP-1, regulates the decision between mitosis and meiosis in the germ line, whereas mouse Notch homologs control neurite outgrowth in differentiated cortical neurons (Berry et al. 1997; Kimble and Simpson 1997; Sestan et al. 1999).

One place where Notch signaling plays an essential, but ill-defined, role is in the process of axis formation in Drosophila, which takes place during the development 
of the oocyte in the ovary (Ruohola et al. 1991). Oogenesis begins at the anterior of the germarium, when a germ line stem cell divides asymmetrically to give rise to another stem cell and a cystoblast, which then undergoes four mitoses with incomplete cytokinesis to generate a cyst of 16 interconnected germ cells (Spradling 1993). As the germ line cyst moves down the germarium, it becomes surrounded by somatically derived follicle cells, which form an epithelium around the cyst, and a stalk of six to eight cells that separates it from the preceding cyst. The first important function of Notch signaling is in migration of the follicle cells, as adjacent egg chambers are often fused in flies that are mutant for a temperature-sensitive allele of Notch under nonpermissive conditions, and this gives rise to giant compound egg chambers that contain multiple germ line cysts in a single follicular epithelium (Ruohola et al. 1991; Xu et al. 1992; Goode et al. 1996a). This Notch-dependent encapsulation of the cyst by the follicle cells is required for the initial polarization of the anterior-posterior (A-P) axis. One of the 16 germ line cells is selected to become the oocyte in region $2 \mathrm{~b}$ and then moves to the posterior of the cyst to generate the first A-P polarity, and this cell rearrangement is driven by $D E$-cadherin-dependent adhesion between the oocyte and the follicle cells that migrate around the posterior (Godt and Tepass 1998; González-Reyes and St. Johnston 1998).

Clonal analysis has revealed that the follicle cells are already divided into two populations in the germarium. One lineage gives rise to the stalk cells and the polar cells, which are a pair of follicle cells at each end of the egg chamber that connect to the stalk, and both cell types never divide once the egg chamber has formed (Tworoger et al. 1999). The rest of the follicle cells derive from a separate lineage and form the epithelium around the cyst (Margolis and Spradling 1995). These cells, which we will refer to as epithelial follicle cells, go through five further rounds of mitosis before switching to the endocycle during stages 7 through 9, in which they become polyploid by cycling through $\mathrm{S}$ phases without intervening cell divisions (Calvi et al. 1998). It is unclear exactly when and where the Notch pathway is required for the migration of the follicle cells around the cysts, but expression of a constitutively active form of Notch results in the formation of extremely long stalks that can contain stalk, polar, or undifferentiated cells (Larkin et al. 1996). Thus, the early function of Notch may be to induce the formation of the stalk by controlling the determination or behavior of the stalk and polar cell populations.

Later in oogenesis, the Notch signaling pathway is required a second time, for the polarization of the A-P axis of the oocyte itself (Ruohola et al. 1991). After the epithelial follicle cells stop dividing at stage 6, they segregate into two subpopulations: the main-body follicle cells in the middle of the egg chamber and 200 terminal follicle cells at each pole (González-Reyes and St. Johnston 1998). The oocyte, which is now at the posterior, then signals through the Gurken/EGFR pathway to induce the adjacent terminal follicle cells to adopt a pos- terior rather than an anterior fate (González-Reyes and St. Johnston 1994, 1995; Roth et al. 1995). At around stage 7 , these posterior follicle cells signal back to the germ line to polarize the microtubule cytoskeleton along the A-P axis of the oocyte, and this defines the A-P axis of the embryo by directing the localization of bicoid mRNA to the anterior of the oocyte and the localization of oskar mRNA and Staufen protein to the posterior (Theurkauf et al. 1992; Clark et al. 1994; Lane and Kalderon 1994; Pokrywka and Stephenson 1995). In addition, this microtubule reorganization triggers the migration of the oocyte nucleus and gurken mRNA from the posterior of the oocyte to the dorsal anterior corner, where Gurken signals for a second time to specify the dorsal-ventral axis of the future embryo (Koch and Spitzer 1983; Neuman-Silberberg and Schüpbach 1993; González-Reyes et al. 1995; Roth et al. 1995). It is not clear where the Notch pathway acts in the process of axis formation, but both Notch ${ }^{\text {ts }}$ and Delta ${ }^{\text {ts }}$ mutations disrupt the polarization of the oocyte at stage 7 (Ruohola et al. 1991). At restrictive temperatures, mutant egg chambers develop a mirror-symmetric A-P axis, in which bicoid mRNA localizes to both poles of the oocyte; oskar mRNA and Staufen protein are transported to the centre; and the oocyte nucleus often fails to migrate from the posterior pole.

Although the phenotypes of Notch ${ }^{\text {ts }}$ and Delta ${ }^{\text {ts }}$ provided the first evidence that the follicle cells signal to polarize the oocyte, the Notch pathway cannot be directly involved in the signaling itself because Notch germ line clones form a normal A-P axis, indicating that the receptor is required in the follicle cell layer and not in the germ line (Ruohola et al. 1991). Furthermore, Delta germ line clones have also been reported to have no effect on axis formation, and several studies have supported the conclusion that Delta is required in the follicle cells as well (Ruohola et al. 1991; Bender et al. 1993; Larkin et al. 1999). Thus, Delta and Notch must act upstream of the signaling to the oocyte and presumably play a role in the patterning of the follicle cells. In wildtype ovaries, Fasciclin III (FasIII) expression becomes restricted to the two polar follicle cells at each end of the egg chamber by stage 4 of oogenesis, but Notch $^{\text {ts }}$ egg chambers contain many more FasIII positive cells at the posterior pole (Ruohola et al. 1991; Goode et al. 1996a). This has led to the suggestion that the defects in A-P axis formation may be a consequence of forming too many posterior polar cells at the expense of the stalk cells (Tworoger et al. 1999). However, these egg chambers also show defects in the specification and patterning of the anterior and posterior terminal populations of epithelial follicle cells (González-Reyes and St. Johnston 1998; Larkin et al. 1999|. Thus, an alternative model is that Notch signaling is required for the formation of the terminal follicle cells, which, unlike the main-body cells, are competent to respond to Gurken by becoming posterior.

Despite the large amount of work on the Notch pathway in oogenesis, the role of Delta/Notch signaling in patterning the follicle cells is still obscure, largely because these studies have only examined females that are 
mutant for Notch $^{\text {ts }}$. Because this allele still retains some activity at restrictive temperatures, the phenotype caused by the complete loss of Notch function is unknown, and these experiments have provided no information on where and when Notch signaling occurs in the follicle cell layer. Here we resolve these questions by generating homozygous mutant clones of null alleles of Notch, Delta, and other components in this pathway. Our results rule out the existing models for the function of Notch in oogenesis and reveal instead that this pathway mediates a novel interaction between the germ line and the soma.

\section{Results}

\section{Notch mutant cells do not adopt the polar cell fate}

To investigate the spatial requirements for Notch in the follicle cell layer, we generated mutant clones marked by the loss of GFP that were homozygous for either a null allele of Notch, $N^{55 \mathrm{e} 11}$, or a strong hypomorphic allele, $N^{\mathrm{X} 81 \mathrm{~K} 1}$, and obtained identical results with both mutations. As one of the clearest phenotypes of Notch ${ }^{\text {ts }}$ is an increase in the number of FasIII positive cells at the posterior of the egg chamber, we first examined the expression of this protein in mutant clones. In wild-type egg chambers, FasIII is expressed in all follicle cells during the germarial and early vitellogenic stages but becomes restricted to the two polar follicle cells at each end of the egg chamber by stage 4 (Fig. 1A). In contrast, follicle cells that lack Notch continue to express FasIII at later stages, and this ectopic expression is cell autonomous and is independent of the position of the clone (Fig. 1B,C). FasIII was always expressed in all mutant cells in the $200 \mathrm{mu}-$ tant clones that we have examined, although it sometimes seems to localize only to the sides of the cell that contact other mutant cells. FasIII is never seen, however, in any heterozygous or wild-type cells except the polar cells. Thus, Notch signaling is required to repress FasIII expression in all epithelial follicle cells of the egg chamber.

The up-regulation of FasIII in Notch mutants has previously been interpreted as transformation to polar follicle cell fate (Ruohola et al. 1991; Goode et al. 1996b; Larkin et al. 1999). As pointed out by Zhang and Kalderon (2000), however, FasIII is also expressed by all follicle cells earlier in oogenesis, and an alternative explanation for this phenotype is that the mutant cells are arrested in an immature state. To distinguish between these possibilities, we analyzed the mutant clones using two more specific polar cell markers that are not expressed by immature follicle cells: the enhancer-trap lines A101 (neur-LacZ; Fig. 1A) and P\{1ArB\}A77.1M3 (Bellen et al. 1989).

Irrespectively of the size or the location of the clones, neither marker is up-regulated in Notch mutant cells (Fig. 1B-D). Furthermore, the mutant clones can contain $>100$ cells, indicating that the cells have gone through multiple divisions after the clone was induced, whereas the polar cells never divide after leaving the germarium. Thus, Notch mutant epithelial follicle cells do not differentiate as polar cells and may arrest instead at a precursor stage.
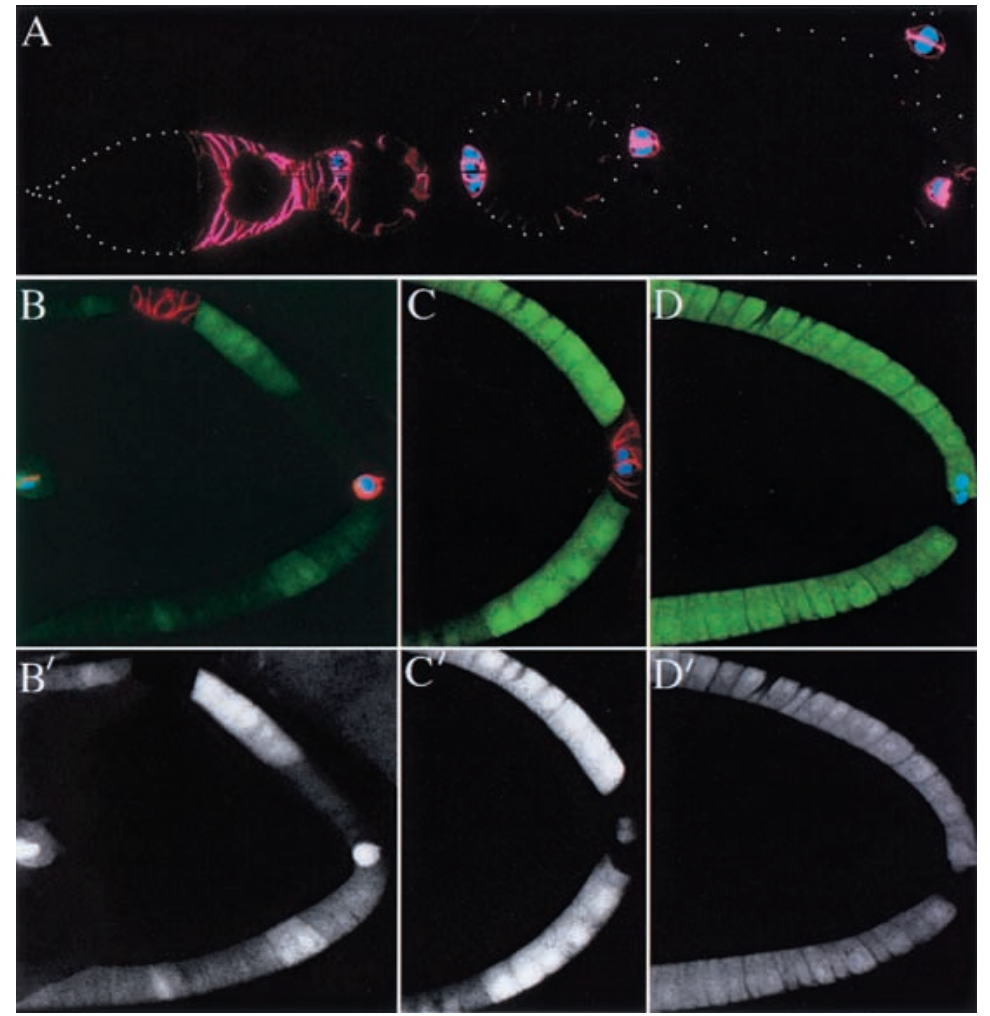

Figure 1. Notch mutant follicle cells are not transformed into polar cells. (A) Expression patterns of Fasciclin III (FasIII) (red) and A101 ( $\beta$-Gal in blue) in a wildtype ovariole. FasIII is expressed in all follicle cells up until stage 3 and then becomes restricted to the polar follicle cells, whereas A101 is expressed only by polar cells. $(B$ and $C)$ Notch mutant cells maintain FasIII expression in a cell autonomous manner, regardless of the position of the clone, but do not express A101. (D) The specific polar follicle cell marker, $P\{1 A r B\} A 77.1 M 3$ (blue), is also not expressed in Notch mutant cells. The lower panels $(B, C)$ show the positions of the clones, which are marked by the loss of GFP (shown in green above). 
To test this hypothesis further, we examined several other enhancer trap lines that are expressed in either immature follicle cells or specific differentiated follicle cell types. In wild type, the $1387 / 16$ enhancer-trap line is expressed in all follicle cells from stage 1 to stage 7 of oogenesis and is then turned off in all cells except the polar and border follicle cells (Fig. 2A). In contrast, $\mathrm{Notch}^{-}$follicle cells express this line cell autonomously until stage 11, the latest stage examined (Fig. 2B,C). We also obtained identical results with another early enhancer trap line, 679/15 (data not shown). Conversely, late markers for differentiated follicle cells are not expressed in mutant clones. For example, the 608/07 and $998 / 12$ lines, which label the posterior terminal follicle cells from stage 8 onward, fail to come on in Notch mutant cells (Fig. 2D,E; data not shown). Finally, when a Notch clone falls in a region where the follicle cells normally undergo morphogenetic movements, these movements do not occur. The stretched follicle cells fail to flatten over the nurse cells; the centripetal cells do not migrate between the oocyte and the nurse cells; and the more posterior follicle cells fail to move over the oocyte (Fig. 2F; data not shown). Taken together, these results indicate that loss of Notch blocks the differentiation of all epithelial follicle cells and causes them to remain as undifferentiated precursors.

This function of Notch in follicle cell differentiation provides a simple explanation for the defects in A-P axis formation that have previously been observed in Notch mutants. The follicle cells at the posterior of the oocyte do not differentiate in the absence of Notch activity, as shown by the lack of posterior marker gene expression (Fig. 2E). As a consequence, these cells cannot signal to polarize the A-P axis of oocyte, and the oocyte nucleus therefore fails to migrate to the dorsal/anterior corner of the oocyte (Fig. 2G).

\section{Notch is required for the differentiation of the polar and stalk follicle cells}

The conclusion that loss of Notch activity does not lead to the formation of extra polar cells raises the question of whether Notch is actually required in the polar follicle cells themselves. We therefore screened for Notch mutant clones in these cells using the polar cell-specific marker A101. In control experiments, we recover clones that include the polar follicle cells in $18 \%$ of the egg chambers, whereas we found no case in which a Notch mutant cell expressed A101 out of 200 egg chambers screened. In addition, we failed to recover any clones in the stalk cells. Thus, the Notch pathway seems to be required for the differentiation of the polar and stalk follicle cells, as well as the epithelial cells.

Several lines of evidence indicate that the encapsulation of the germ line cysts depends on the polar and stalk cells (Larkin et al. 1996, 1999). Consistent with this, adjacent egg chambers are often fused in ovaries containing Notch mutant clones, and the polar cells and stalk cells are always absent in these cases (Fig. 3A). In contrast, large Notch mutant clones in the epithelial follicle cell
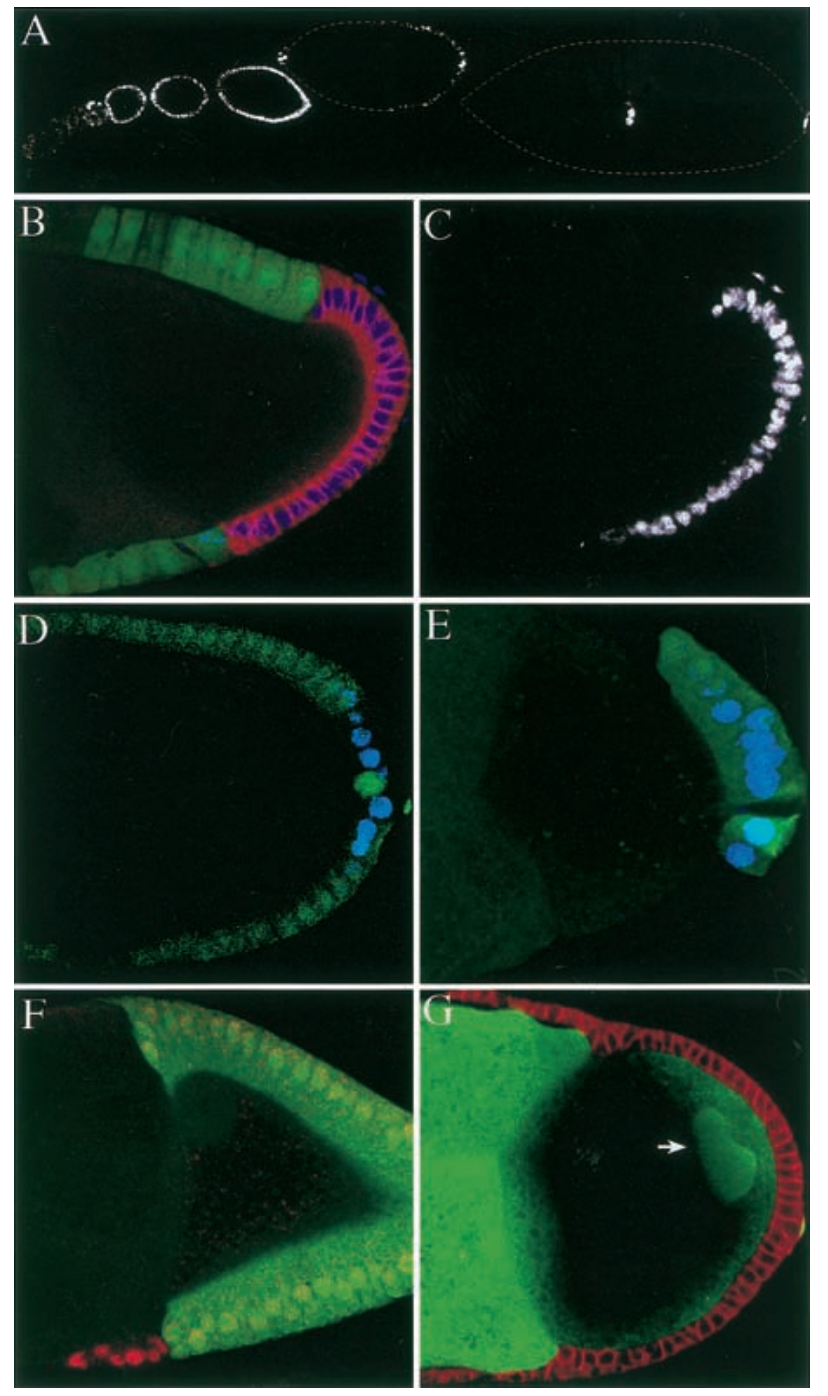

Figure 2. Follicle cells lacking Notch activity do not differentiate. (A) The 1387/16 enhancer trap line is expressed by all immature follicle cells and then becomes restricted to the polar and border cells at stage 7. (B) Expression of Fasciclin III (FasIII) (red) and 1387/16 (blue) in a stage 10 egg chamber containing a Notch mutant follicle cell clone marked by loss of GFP (green). The mutant cells continue to express both FasIII and 1387/16. (C) shows the expression pattern of 1387/16 in this egg chamber to highlight the cell autonomy of Notch mutant phenotype. This marker is only expressed in the mutant cells, which lack GFP in $B .(D, E)$ The 608/07 enhancer-trap line (blue) is normally expressed in the posterior follicle cells from stage 8 onward $(D)$ but is not expressed in Notch mutant cells (marked by loss of GFP in $E) .(F)$ A stage 10 egg chamber containing a Notch mutant clone marked by the persistent expression of 1387/16 (red) and loss of GFP (green). Although the wild-type follicle cells have moved posteriorly to cover the oocyte, the mutant cells have failed to migrate. $(G)$ A large posterior Notch mutant clone in the follicle cell layer, which has blocked the polarization of the oocyte. The oocyte nucleus (arrow) has failed to migrate to the dorsal/anterior corner and remains at the posterior pole. This image was taken using a higher laser power than the others in this figure to visualise the oocyte nucleus. 


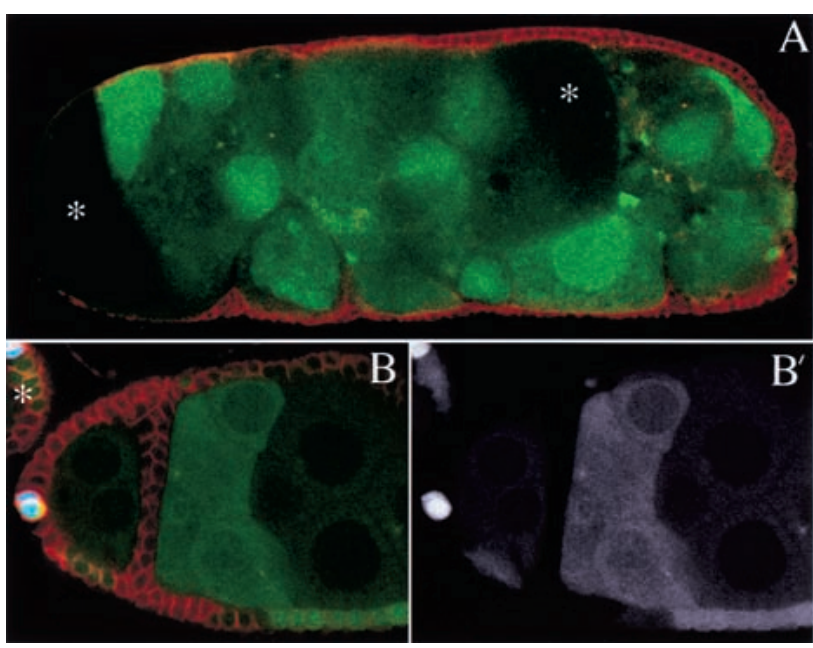

Figure 3. Notch is required the differentiation of the polar cells and egg chamber encapsulation. (A) A fused egg chamber in which all of the follicle cells are mutant for Notch. The mutant follicle cells have not migrated between the germ line cysts, resulting in the formation of a compound egg chamber in which a single follicular epithelium surrounds several cysts. The oocytes of two of the fused germ line cysts are visible in this confocal section (asterisks). (B) A large Notch mutant follicle cell clone marked by loss of GFP expression $\left(B^{\prime}\right)$ in an ovary in which the polar cells are labeled by A101 (blue). Although the mutant follicle cells have formed an epithelium between the two egg chambers, the polar cells are absent, as shown by the lack of A101 expression, and the stalk has failed to form. Note that polar follicle cells at the anterior pole of the younger egg chamber are not mutant $(B)$ and express A101. This egg chamber was able to form a stalk and did not fuse with the younger egg chamber on the left (asterisk). All clones are double marked by absence of GFP (green) and Fasciclin III expression (red).

layer have no effect on cyst encapsulation (Figs. 2B,C; $4 \mathrm{~A})$. In rare cases, adjacent egg chambers are only partially fused, with a single or double layer of follicle cells between them (Fig. 3B,B). In these examples, the boundary between the partially fused cysts is covered by mutant epithelial follicle cells, but there are no A101 positive cells where the polar follicle cells would be expected to lie. In contrast, adjacent cysts are separated normally when the polar cells are wild type but the epithelial cells are mutant, as seen at the anterior of the younger cyst in Figure 3, B and 3Bprime; . These results are consistent with a model in which Notch pathway mutants disrupt the encapsulation of the germ line cysts because Notch signaling is required for the differentiation of the polar and stalks cells that mediate this process.

\section{Notch is required for the epithelial follicle cells} to exit the mitotic cell cycle

The differentiation of the epithelial follicle cells is first apparent at stage 7 and occurs after the cells have exited the mitotic cell cycle and entered the endocycle to become polyploid. Because Notch mutations arrest these cells at the precursor stage, we examined whether they also prevent this switch in the cell cycle, by analyzing the division patterns of mutant clones. At later stages of oogenesis, mutant clones always contain many more cells than the wild-type twin spot clones that were induced at the same time (Fig. 4A). The mutant clones still occupy the same area as the twin spot clones, however, because the Notch mutant cells are much smaller than their wild-type siblings. Thus, the loss of Notch causes the cells to go through extra cell divisions, without a corresponding increase in growth rate, to generate a larger number of smaller cells.

To determine when these extra divisions occur, we used an antibody against the phosphorylated form of the Histone- $\mathrm{H} 3$, which labels cells in late $\mathrm{G}_{2}$ and mitosis but not cells in the endocycle (Wei et al. 1999). Before stage 6 , there is no obvious difference between the frequency of mitoses in wild-type and mutant cells. Wild-type cells never stain for phospho-Histone H3 after stage 6, however, whereas mutant cells continue to divide up until stages 10B or 11 (Fig. 3B). Furthermore, mutant cells have a lower DNA content and smaller nuclei than do wild-type cells, as revealed by OliGreen staining (Fig. 3C). These results indicate that Notch mutant cells fail to switch from the mitotic cell cycle to the endocycle, and carry on dividing instead of becoming polyploid.

\section{Mutations in other components of the Notch signaling pathway display similar defects in the follicular epithelium}

To determine whether Notch signaling in the follicle cells occurs through the canonical pathway described in other cell types, we generated follicle cell clones of mutants in downstream components in this signal transduction cascade. Follicle cells clones of null allele of presenilin and $\mathrm{Su}(\mathrm{H})$ give rise to the same array of phenotypes as Notch mutant clones. Large clones that presumably include the polar cell precursors lead to fusions between adjacent egg chambers, whereas mutant epithelial follicle cells up-regulate FasIII in a cell autonomous manner and continue to divide after stage 6 (Fig. 5A,B). When the mutant clones include the follicle cells at the posterior of the egg chamber, these cells cannot signal to polarize the oocyte, and the germinal vesicle therefore often fails to migrate and remains at the posterior pole (Fig. $5 A, B)$. Thus, Notch signaling in the follicle cells seems to act through the standard pathway, in which Notch undergoes a presenilin-dependent cleavage to generate Notch $^{\text {intra, }}$, which then translocates to the nucleus to regulate transcription in association with $\mathrm{Su}(\mathrm{H})$ protein.

In lateral inhibition, Notch ${ }^{\text {intra }}$ and $\mathrm{Su}(\mathrm{H})$ protein activate the transcription of the genes of the $E(s p l)$ complex, which then mediate the response to Notch by repressing proneural gene expression. In contrast, follicle cell clones of a deficiency that removes all of the $E$ (spl) complex transcription factors have no detectable phenotype. Mutant cells do not up-regulate FasIII and stop mitosis at stage 6, as in wild type (Fig. 5C). Furthermore, mutant posterior cells still signal to polarize the oocyte, because GFP-Staufen localizes normally to the posterior 
Figure 4. Notch mutant follicle cells fail to switch from the mitotic cell cycle to the endocycle. $(A)$ Surface view of a stage $10 \mathrm{~b}$ egg chamber containing a Notch mutant clone and a wild-type twin spot clone, in which the cell outlines are revealed by Rhodamine -Phalloidin staining of the actin cytoskeleton (red). The twin spot clone expresses higher levels of GFP (green) because it contains two copies of the transgene, whereas the mutant clone is marked by the absence of GFP. The two clones are similar in size, but the mutant clone contains many more cells that are much smaller than the wild type ones. (B) A stage 10B egg chamber containing a Notch mutant clone stained for phosphoHistone-H3 (red) and $\alpha$-tubulin (blue). One of the mutant cells is $\mathrm{P}-$ Histone-H3 positive and has formed a mitotic spindle (inset), indicating that the mutant cells continue to divide after stage 5. (C) OliGreen DNA staining of the border between a Notch mutant clone and wild-type cells. The mutant cells are marked by the up-regulation of FasIII expression (red), and have smaller nuclei with a lower DNA content, indicating that they have not become polyploid.
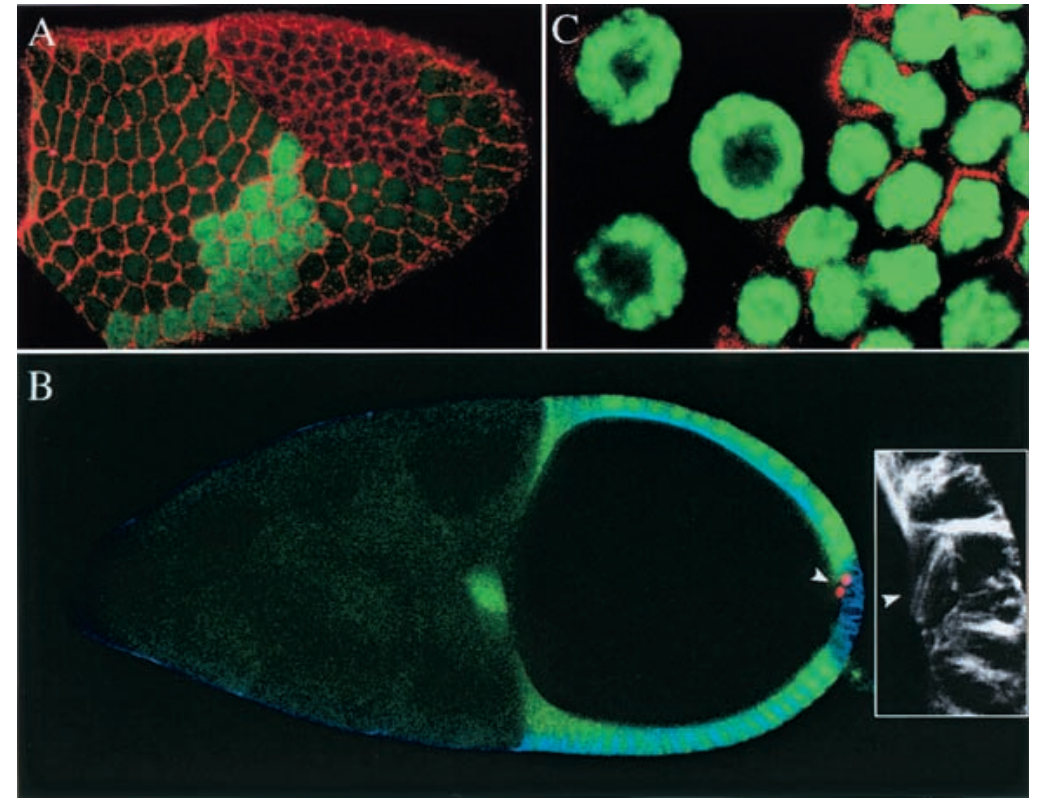

pole of the oocyte in the presence of large posterior follicle cell clones. Thus, Notch signaling in the follicle cells does not require the transcription factors of the E(spl) complex, indicating that Notch ${ }^{\text {intra }}$ and $\mathrm{Su}(\mathrm{H})$ exert their effects by regulating other target genes.

The final component of the Notch pathway that we examined is Hairless, which acts as a negative regulator of Notch signaling by binding to $\mathrm{Su}(\mathrm{H})$ protein (Brou et al. 1994; Schweisguth and Posakony 1994; Bang et al. 1995; Schweisguth and Lecourtois 1998). Clones of Hairless cause a large expansion of the stalks between adjacent egg chambers (Fig. 5D). This phenotype is identical

Figure 5. Notch signaling in the follicle cells requires presenilin and $\mathrm{Su}(\mathrm{H})$ but not $\mathrm{E}(\mathrm{spl})$. (A) A stage 9 egg chamber containing a large $\mathrm{Su}(\mathrm{H})$ mutant clone. Loss of $\mathrm{Su}(\mathrm{H})$ in the follicle cells produces the same phenotype as loss of Notch: The mutant follicle cells up-regulate Fasciclin III (FasIII) (red) and adjacent egg chambers fuse, and the oocyte is not polarized, as shown by the failure of the oocyte nucleus to migrate from the posterior pole (arrow). (B) A stage 10 egg chamber containing two psn mutant follicle cell clones, marked by loss of nuclear GFP (green). The mutant cells up-regulate Fas III (red) and remain mitotically active, as shown by the P-Histone H3 staining (arrowhead). The oocyte nucleus (arrow) has not migrated to the anterior, indicating that the posterior follicle cell clone has disrupted the polarization of the oocyte. $(C)$. A posterior clone of E(spl) mutant cells in a stage 10 egg chamber that has been stained for FasIII (red) and P-Histone-H3 (blue) and expresses GFP-Staufen in the germ line (green). The mutant cells, which are marked by loss of nuclear GFP (green), do not express FasIII or P-Histone-H3, and the oocyte is polarized normally, because GFP-Staufen localizes to the posterior pole. The polar follicle cells are not mutant and show the normal expression of FasIII. (D) Hairless mutant follicle cells form very long stalks, containing more than the usual number of 7 and 8 follicle cells.
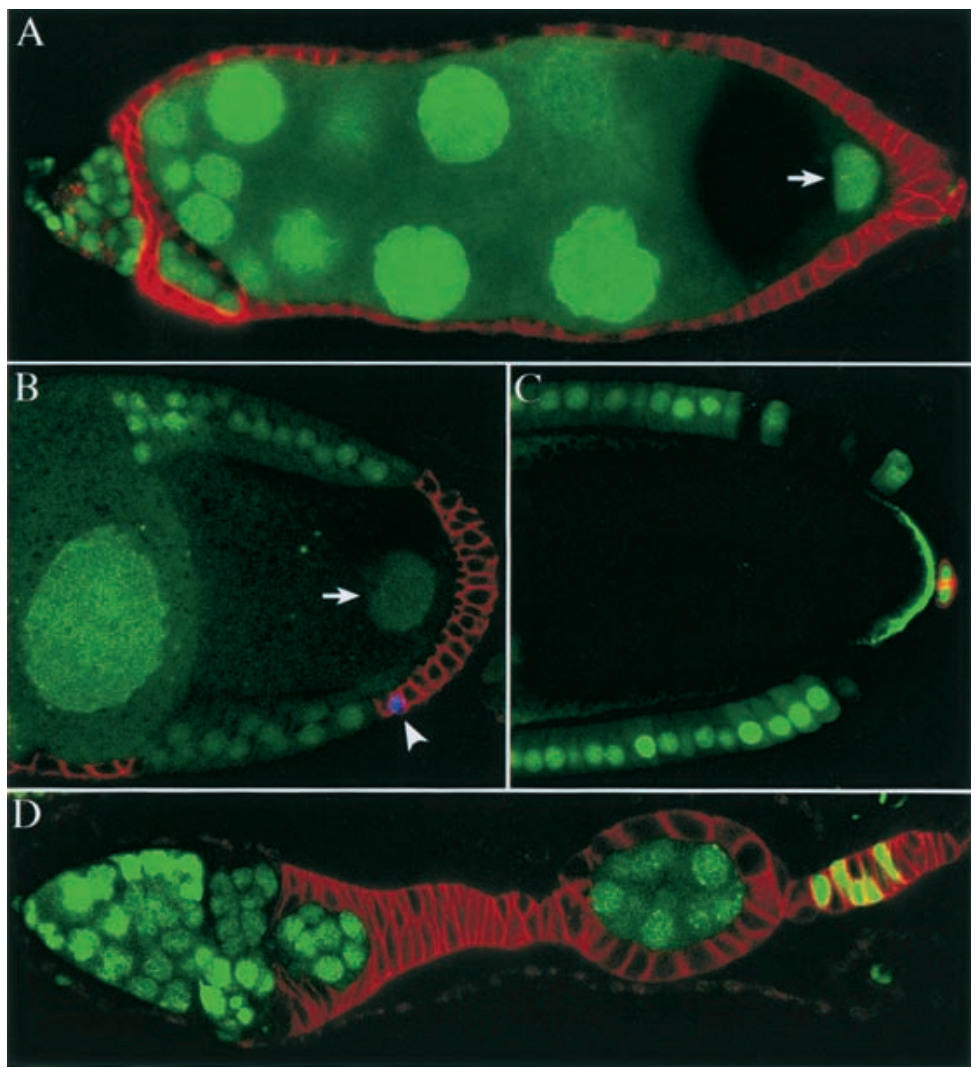


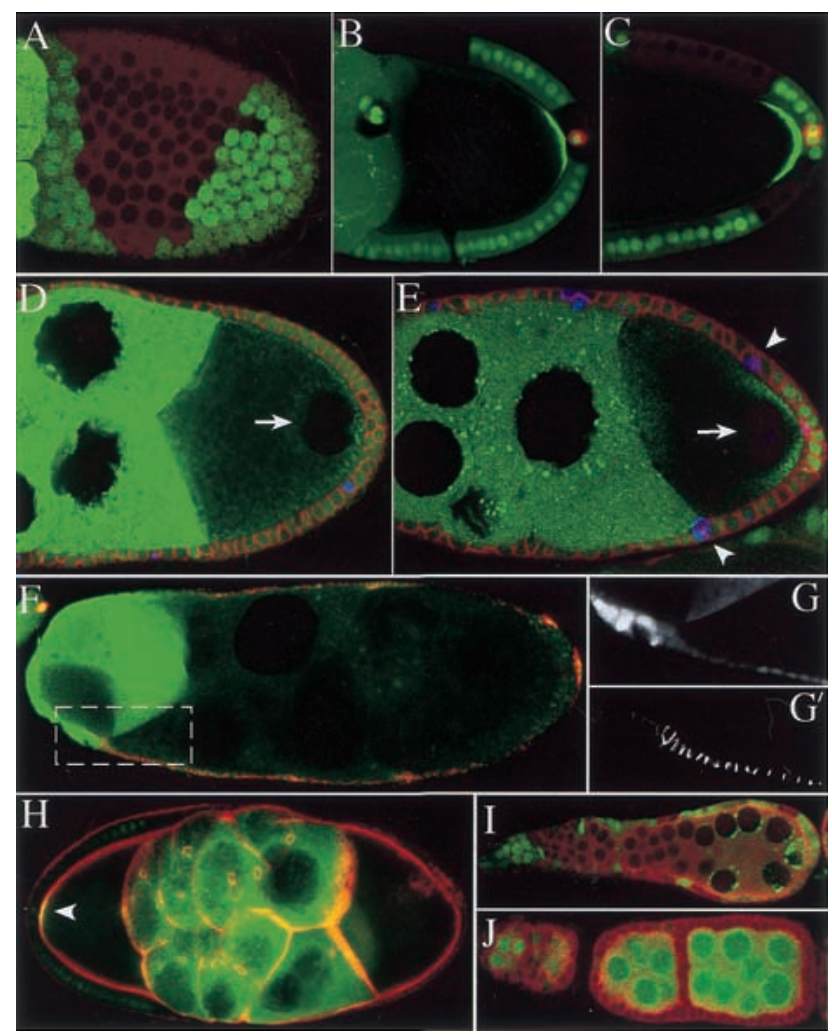

to that produced by the expression of constitutively active forms of Notch, consistent with the model in which loss of Hairless leads to overactivation of the signaling pathway (Larkin et al. 1996). In contrast, Hairless clones in the epithelial follicle cells have no discernable phenotype. Thus, Hairless protein seems to be required to inhibit Notch signaling in the polar and stalk cell lineage, but it is not necessary for the development of the epithelial cells.

\section{Delta activity is required in the germ line}

The demonstration that Notch signaling is required for the differentiation of all follicle cells implies that every cell must be exposed to a DSL ligand that can activate Notch, and this raises the question of where these signals are produced. It has previously been reported that temperature-sensitive alleles of the DSL ligand, Delta, cause many of the same phenotypes as Notch ${ }^{\text {ts }}$, whereas germ line clones of Delta do not, suggesting that Delta is required in the somatic follicle cell layer for the activation of Notch (Ruohola et al. 1991; Larkin et al. 1999). We therefore generated follicle cell clones of a strong loss of function allele, $D l^{\mathrm{M} 1}$, to map which cells produce this signal. Although we obtained occasional partial fusions between the adjacent egg chambers, which we discuss below, mutant clones cause no other obvious defects in the follicular epithelium, even when they include several hundred cells. In particular, follicle cells in the vicinity of the mutant clones never show any of the phe-
Figure 6. Delta is required in the germ line. $(A, B)$ Delta mutant follicle cells clones, stained for Fasciclin III (FasIII) (red) and P-Histone-H3 (blue). The mutant cells, which are marked by loss of nuclear-GFP (green), do not up-regulate FasIII or go through extra divisions. GFP-Staufen has been expressed in the germ line of the egg chamber in $B$ and localizes normally to the posterior pole of the oocyte, indicating that Delta mutant posterior follicle cells can still signal to polarize the oocyte. $(C)$ A posterior follicle cell clone mutant for both Delta and Serrate. The mutant cells develop normally and do not up-regulate FasIII. $(D, E)$ Stage $10 \mathrm{~A}$ egg chambers in which the germ line is mutant for Delta (marked by the loss of green nuclear-GFP expression), but most of the follicle cells are wild type.The wildtype follicle cells (nuclear-GFP positive) up-regulate FasIII (red) and are positive for P-Histone-H3 (blue, arrowheads), indicating that they are still dividing. In both cases, the oocyte nucleus (arrows) has failed to migrate to the anterior, because the oocyte is not polarized. The green staining in the cytoplasm of the germ cells is GFP-Staufen, which does not localize as a tight crescent to the posterior of the oocyte. (F) Fused egg chamber containing a wild-type germ line cyst (bright green GFP expression, left) and a Delta mutant germ line cyst (no GFP, right). The follicle cells in contact with the Delta mutant germ cells do not differentiate, and express high levels of FasIII (red), whereas those in contact with wild-type germ cells down-regulate FasIII. $\left(G, G^{\prime}\right)$ A magnified view of the boxed region of $(\mathrm{F})$ showing $\mathrm{GFP}(\mathrm{G})$ to mark the border between the wild-type and mutant gem cells, and Fas III expression $\left(G^{\prime}\right)$. (H) A fused egg chamber containing a wild-type (left) and a Delta mutant germ line cyst (right) that express GFP-Staufen in the germ line (cytoplasmic green signal) stained with Rhodamine Phalloidin (red) to label actin. GFPStaufen localizes to the posterior pole of the wild-type oocyte, but is not localized in the mutant oocyte, because the surrounding follicle cells have not differentiated. (I) Large Delta germ line clones (marked by the loss of nuclear GFP) lead to the fusion of multiple germ line cysts. (The green signal in the cytoplasm corresponds to GFP-Staufen). (J) Large Delta follicle cell clones lead to the loss of the stalks between adjacent wildtype cysts, but do not cause a complete fusion.

notypes associated with loss of Notch signaling: The cells do not up-regulate FasIII expression or go through extra divisions, and posterior clones have no effect on the polarity of the oocyte, as shown by the wild-type localization of GFP-Staufen to the posterior pole (Fig. $6 \mathrm{~A}, \mathrm{~B})$. One explanation for this lack of a phenotype could be that Delta functions redundantly with Serrate, the other DSL ligand in Drosophila. To test this possibility, we generated follicle cell clones homozygous for both Ser ${ }^{\mathrm{RX} 82}$ and $D 1^{\text {rev10 }}$ (Micchelli et al. 1997), which are both null alleles, and again we observed none of these phenotypes (Fig. 6C). Thus, neither of the DSL ligands is required in the follicle cell epithelium, suggesting that the signal that activates Notch in these cells is produced elsewhere.

Delta and Serrate are both transmembrane proteins that signal to Notch in adjacent cells, and the only other cells that directly contact the follicle cells are the germ line cells of the cyst. We therefore re-examined the requirement for Delta in the germ line by generating germ line clones, using the dominant female sterile mutation ${ }_{\text {ovo }}{ }^{\mathrm{D}}$ (Chou and Perrimon 1996). Contrary to the previous report, these clones cause severe encapsulation de- 
fects, leading to the complete fusion of adjacent egg chambers into a single large compound egg chamber that contains multiple germ line cysts (Fig. 6I). Our previous results have shown that Notch signaling is only required in the polar and stalk cell lineage for cyst encapsulation, and this phenotype therefore suggests that Delta signals from the germ line early in oogenesis to activate Notch in some or all of these cells.

The massive disruption of oogenesis caused by egg chamber fusions in Delta mutant clones generated using ${ }_{O V O}{ }^{\mathrm{D}}$ precludes the analysis of the behavior of the epithelial follicle cells, and we therefore induced smaller clones that were marked by the loss of nuclear GFP (Luschnig et al. 2000). When the germ cells of a single egg chamber are mutant for Delta, the wild-type follicle cells that surround them show all of the phenotypes associated with loss of Notch activity in the follicle cell layer. The whole epithelium up-regulates FasIII expression, and the cells continue to divide well beyond stage 6 , as shown by phospho-Histone H3 labeling (Fig. 6D,E). Furthermore, these egg chambers show a strong and highly penetrant oocyte polarity defect, as the oocyte nucleus remains at the posterior pole of the cell in 56 out of 61 cases (Fig. 6D,E). Thus, Delta is required in the germ line for the activation Notch in all epithelial follicle cells, and signals to control both the cell cycle and differentiation of these cells.

Individual egg chambers bearing Delta germ line clones usually fuse with an adjacent wild-type one (62/ 89) to form a mosaic egg chamber in which a single follicular epithelium surrounds both wild-type and mutant germ cells. In these cases, the follicle cells that surround the mutant germ cells fail to turn off a marker for immature follicle cells at the appropriate stage, whereas the cells in contact with wild-type germ cells develop normally (Fig.6F-G). These hybrid egg chambers also demonstrate the requirement for Delta signaling in A-P axis formation. In the wild-type cyst, GFP-Staufen localizes normally to the posterior pole of the oocyte because the overlying follicle cells differentiate and signal, whereas the A-P axis is not polarized in the mutant oocyte because of the lack of a polarizing signal from the undifferentiated follicle cells at the posterior pole (Fig. 6H).

Although Delta germ line clones reproduce all of the phenotypes caused by loss of Notch signaling in the follicle cells, Delta must also signal between follicle cells during cyst encapsulation, because large mutant follicle cell clones cause partial fusions between adjacent cysts of wild-type germ cells (Fig. 6J). In these cases, the cysts are still surrounded by follicle cells, but the stalk between them is completely absent. This observation is consistent with the fact that the stalk cells are the only follicle cells that do not contact the germ cells directly and therefore cannot respond to Delta in the germ line. The most likely source of this signal is the polar cells, because the loss of Delta in the epithelial cells produces no phenotype. This suggests that there is a relay of Delta signaling during stalk formation, in which the germ line signals to induce Delta in the polar cells, which in turn signal to induce the stalk cells.

\section{Timing of Delta/Notch signaling}

Our results indicate that Delta signals from the germ line to activate Notch in the somatic follicle cells twice during oogenesis: once in the germarium to induce the differentiation of the polar/stalk cell lineage and then later to induce the differentiation of the epithelial follicle cells. To see if this correlates with expression of the two proteins, we stained wild-type ovaries for Notch and Delta. Notch protein is expressed from the germarium up to stage 7 and localizes to the apical membrane of the follicle cells, in close contact with the germ line. This apical localization disappears after this stage, leaving behind a faint punctate cytoplasmic staining (Fig. 7A,B; Xu et al. 1992). Delta protein expression shows a different but complementary pattern of expression. Delta is expressed by the germ line and the soma, but it is particularly abundant in the nurse cells and oocyte, where it seems to accumulate at the plasma membrane, and in large particles in the cytoplasm (Fig. 7C; Bender et al. 1993). Delta protein is present at low levels during early oogenesis but increases in abundance from stage 5 onward to reach its highest levels at stage 7 , right at the time when Notch protein disappears from the apical membrane of follicle cells. This suggests that there is a burst of Delta signaling during stages 5 through 7, which removes most Notch protein from the membrane, either because the receptor is cleaved on binding to Delta or because Notch is down-regulated in response to the activation of the pathway, as is the case for the secondary mesenchyme cells of sea urchin embryos (McClay et al. 2000). In either case, the loss of Notch protein from the follicle cell membranes should depend on Delta signaling, and we therefore examined the distribution of Notch protein in Delta germ line clones, using antibodies directed against the extracellular and intracellular portions of the receptor. Both antibodies reveal that Notch is not down-regulated in Delta mutant egg chambers. This is particularly clear in chimaeric egg chambers that contain both wild-type and mutant germ cells, where Notch remains associated with the apical membrane of all follicle cells in contact with the mutant germ cells but disappears from those overlying the wild-type germ line cells (Fig. 7D,E). This suggests that the Delta signals to the epithelial follicle cells at around stages 5 to 7, which coincides with when these cells cease dividing and start to differentiate.

\section{Discussion}

Our results lead to a simple model for the function of the Notch pathway in oogenesis in which Delta signals from the germ line twice to control the differentiation of the two different lineages of follicle cells. The first signal is sent in the germarium, where Delta is required in the germ line for the encapsulation of the cysts by the somatic follicle cells in region $2 \mathrm{~b}$. The Notch receptor is only required in the stalk and polar cells at this stage and not in the epithelial follicle cells, indicating that Delta regulates follicle cell migration by controling the behav- 

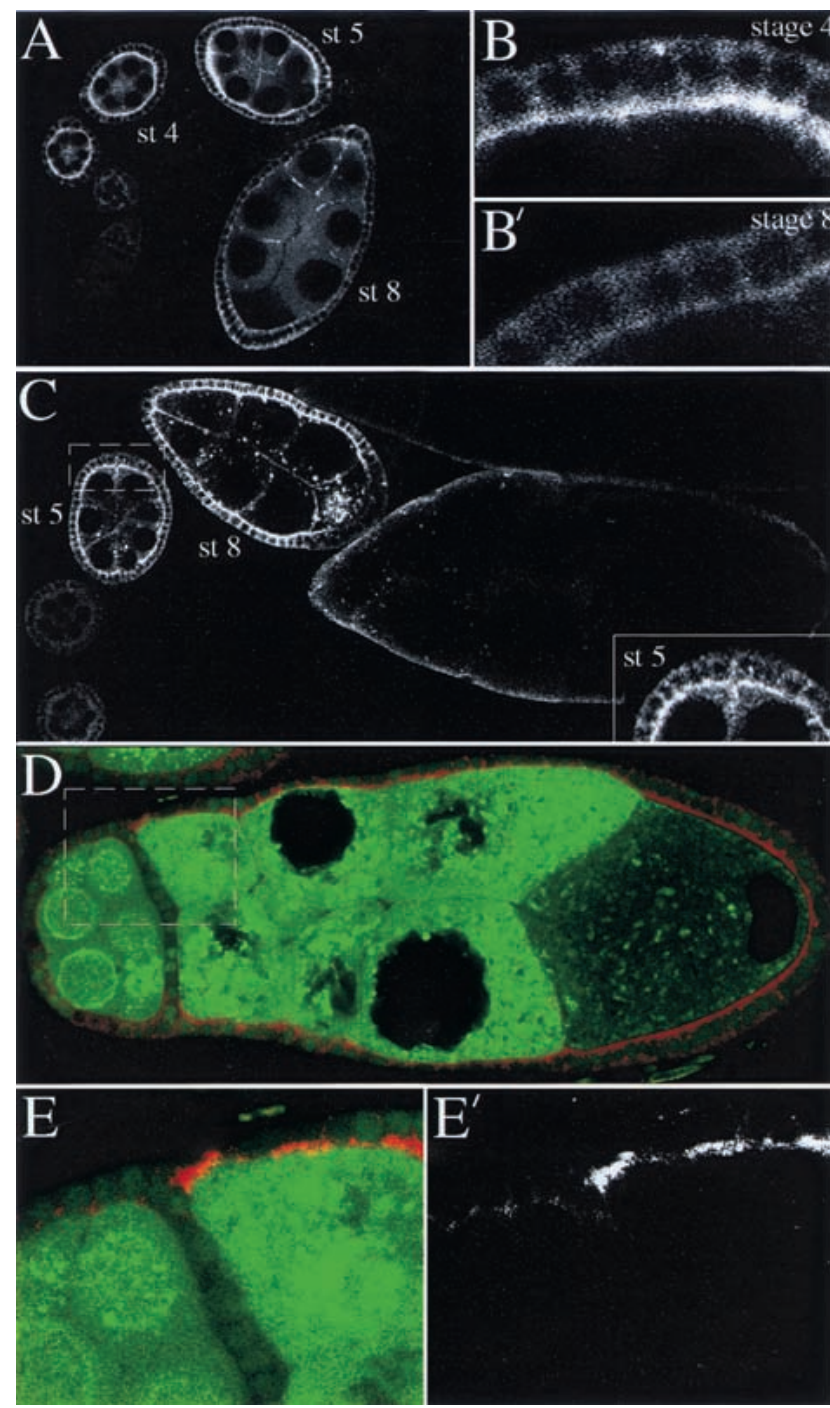

Figure 7. Localization of Notch and Delta proteins during oogenesis. (A) Wild-type ovariole stained with an antibody against the intracellular portion of Notch protein. Notch accumulates in the apical membranes of the follicle cells during stages 3 through 6 of oogenesis, but is down-regulated after this stage. (B, $\left.B^{\prime}\right)$ show a higher magnification view of the follicle cells at stages 4 and 8 to the show the disappearance of Notch from the apical membrane. $(C)$ Wild-type ovariole stained with an antibody against Delta. Delta protein is expressed in both the germ line and the follicle cells, and becomes highly enriched at the surface of the germ cells during stages 5 through 7 . The inset shows a higher magnification view of a stage 5 egg chamber to highlight the localization of the germ line-expressed Delta protein. $(D)$ Notch staining of a partially fused egg chamber containing a wild-type and a Delta mutant germ line cyst expressing GFP-Staufen. Notch protein remains associated with the apical membrane of the follicle cells in contact with the Delta mutant germ cells, but disappears from the cells that surround the wild-type cyst (left, marked by nuclear -GFP; green). (E, E') Higher magnification view of the border between the wild-type and mutant cysts in (boxed region in $D$ ) to show the maintenance of apical Notch protein $\left(E^{\prime}\right)$ in the follicle cells in contact with the mutant germ cells. ior of the stalk/polar cell lineage. The direct target of this germ line signal is presumably the polar cells, as these cells are in contact with the germ cells, and the polar cells may then respond by expressing Delta themselves to relay this signal to the adjacent stalk cells.

The second round of Delta signaling occurs between stages 5 to 7 of oogenesis and regulates the behavior of other follicle cell population, the epithelial cells. Loss of Delta from the germ line or of Notch, $p s n$, or $\mathrm{Su}(\mathrm{H})$ from the epithelial cells themselves blocks the differentiation of these cells and causes them to arrest in an immature state. Furthermore, mutant cells do not switch from the mitotic cell cycle to the endocycle at stage 6 and continue to divide for the rest of oogenesis. Thus, Delta signals to all follicle cells during the course of oogenesis and is required for the differentiation of every follicle cell type.

One question raised by these results is why the epithelial cells do not respond to Delta in germarium, whereas the polar and stalk cells do, as the epithelial cells also contact the germ line. A possible explanation for this difference in competence is that the stalk and polar cells express high levels of fringe, which encodes a glucosyltransferase that modifies Notch to make it more sensitive to Delta signaling (Bruckner et al. 2000; Jordan et al. 2000; Moloney et al. 2000; Munro and Freeman 2000; Zhao et al. 2000). Thus, these cells can presumably respond to the low levels of Delta in the germarium, whereas the epithelial cells, which do not express fringe early in oogenesis, can only respond when Delta is expressed at much higher levels at stages 5 to 7 .

The role of the Notch pathway in oogenesis has received considerable attention because it is required for the polarization of the A-P axis of the oocyte. Based on the analysis of the $\mathrm{Notch}^{\text {ts }}$ allele, two conflicting models have been proposed to explain the role of this pathway in axis formation. The observation that extra posterior cells express FasIII in mutant females has been interpreted to show that a reduction in Notch activity leads to a transformation of stalk cells into polar cells, which somehow disrupts the signaling to polarize the oocyte (Ruohola et al. 1991; Bender et al. 1993). Our results using more specific markers rule out this model, however, because they reveal that the extra FasIII expressing cells are not polar cells but epithelial cells that are arrested in an undifferentiated state. In contrast, the second model proposes that Notch is required for the determination and patterning of the terminal populations within the epithelial follicle cell layer and is based on the fact that the expression of both anterior and posterior terminal follicle cell markers is disrupted in Notch ${ }^{\text {ts }}$ (González-Reyes and St. Johnston 1998; Larkin et al. 1999). The clonal analysis is also inconsistent with this role for the pathway in patterning specific follicle cell types, because the loss of Notch causes an identical phenotype in all epithelial cells, regardless of their position. Nevertheless, our results do agree with the conclusion that the A-P axis defect is caused by the failure of the terminal cells to differentiate. If a mutant clone includes the posterior terminal cells, these undifferentiated cells are unable to 
respond to Gurken by becoming posterior and therefore do not signal to polarize the oocyte. The requirement for Notch in follicle cell differentiation therefore provides a straightforward explanation for the defects in axis formation, and can account for all of the other phenotypes that have been reported for Notch ${ }^{\text {ts }}$.

\section{A novel role for Notch in cell cycle control}

The function of Notch in oogenesis is very different from its well-characterized role in lateral inhibition, where it acts to inhibit the determination and differentiation of the cells in which it is activated (Greenwald 1998; Artavanis-Tsakonas et al. 1999). In contrast, Notch signaling in the follicle cells promotes differentiation rather than inhibiting it, as neither polar, stalk, nor epithelial cells are formed in mutant clones. Although we do not know the fate of the mutant stalk and polar cell precursors, loss of Notch signaling prevents the epithelial cells from switching from the mitotic cell cycle to the endocycle. Thus, the primary target of Notch signaling may be to control proliferation, which then allows the cells to differentiate. In general, cells stop dividing when they differentiate, but the relationship between these two processes is not well understood, and it is also possible that Notch regulates both events in parallel or that it induces differentiation, which in turn blocks division (Myster and Duronio 2000).

Notch signaling has been shown to control the cell cycle in several other contexts but usually acts to promote mitosis instead of inhibiting it. For example, activation of the C. elegans Notch homolog GLP-1 maintains germ cells in mitosis, while constitutive activation of Drosophila Notch causes the overproliferation of imaginal disc cells (Berry et al. 1997; Kimble and Simpson 1997; Go et al. 1998; Baonza and Garcia-Bellido 2000). Notch signaling has been reported to play a role in inhibiting mitosis in one other case. In conjunction with Wingless, activation of Notch at the dorsal-ventral boundary of the imaginal wing disc arrests the cell cycle at the $\mathrm{G}_{1}$ phase, to form a zone of nonproliferating cells (ZNC; Johnston and Edgar 1998). However, this function of Notch differs from its role in the follicle cells in several respects. First, the cell cycle arrest in the wing disc is absolutely dependent on Wingless signaling, which does not seem to be required in the epithelial follicle cell layer (Forbes et al. 1996). Second, Notch causes $G_{1}$ arrest in the ZNC by inhibiting the expression of the achaetescute complex, a function that presumably requires the $E(s p l)$ complex genes. In contrast, the E(spl) complex is not required in the follicle cells, where Notch must signal through other target genes. Finally, the follicle cells do not arrest in $G_{1}$ but continue to cycle through $S$ phase to become polyploid. Thus, signaling in the follicle cells represents a new activity for Notch pathway, in which Notch signaling leads to the repression of the $M$ phase of the cell cycle but allows cells to continue to replicate their DNA.

The development of a complex structure requires a high degree of coordination between the cells that com- pose it, and the relative timing of development in different cell types must therefore be regulated. The discovery that Delta activity during oogenesis is germ-line dependent provides a simple mechanism for coordinating the development of the germ line and somatic cells of the egg chamber. The up-regulation of Delta in the germ cells during stages 5 through 7 controls when Notch is activated in the epithelial follicle cells and can therefore regulate the timing of the development of these cells, by determining when they cease proliferation and differentiate. Furthermore, this signal probably also synchronizes the development of the whole follicle cell layer, because the germ line syncytium can signal to all epithelial follicle cells at the same time. This function is remarkably similar to the proposed role of Delta/Notch signaling in the presomitic mesoderm, where it is thought to synchronize the cell cycle in adjacent cells so that they coordinately differentiate into somites (Jiang et al. 2000). It will therefore be interesting to see how universal this mechanism is and to know if other examples of Notch signaling display a similar correlation between cell fate determination and cell cycle progression.

The germ line has previously been shown to signal to the soma twice during oogenesis to control the patterning of the follicle cell layer. At stages 6 and 7, Gurken signals from the germ line to activate the Drosophila EGF receptor in terminal follicle cells to induce them to adopt a posterior fate, and at stages 9 and 10 Gurken signals to main-body follicle cells to induce them to become dorsal (Neuman-Silberberg and Schüpbach 1993; González-Reyes et al. 1995; Roth et al. 1995). Our results show that the germ line plays an even greater role in the development of the follicle cells, because Delta also signals twice from the germ line to Notch in the soma. These two pathways have complementary effects on the follicle cells: Gurken/DER signaling exerts spatial control, whereas Delta/Notch signaling seems to exert temporal control. In other respects, these processes are rather similar. Like Gurken, Delta signals to two distinct follicle cell types at different stages, in this case the polar/stalk cells and then the epithelial cells. Furthermore, the competence of the follicle cells to respond to both signals appears to be regulated. The epithelial cells do not respond to Delta when it signals to the polar and stalk cells, perhaps because they lack fringe, and the main-body follicle cells cannot respond to Gurken when it signals to the terminal cells (González-Reyes and St. Johnston 1998; Larkin et al. 1999). Finally, it is worth noting that Gurken signaling is absolutely dependent on Delta signaling, because the follicle cells cannot adopt the correct cell fate in response to Gurken unless they have already been rendered competent to differentiate by Delta.

\section{Oogenesis as a model system for Delta/Notch signaling}

Drosophila oogenesis provides an excellent system in which to analyze the Notch signaling pathway for several reasons. First, Delta signals to activate Notch in a large number of follicle cells at the same time, because 
1000 epithelial follicle cells receive the second signal during stages 5 through 7 . Second, the epithelial cells constitute one of the rare examples where the downregulation of Notch in response to Delta can be observed directly, and this allows one to see when and where signaling takes place. Finally, the cells that send the signal are clearly distinct from the cells that receive it, because Notch is not required in the germ line, nor Delta in the epithelial cells. The germ cells form a separate lineage from the rest of the organism very early in embryogenesis, and it is therefore straightforward to determine whether other genes in the pathway act in the signaling or responding cells. Two other neurogenic genes, egghead and brainiac, have previously been shown to produce Notch-like phenotypes during oogenesis (Goode et al. 1992; Goode et al. 1996a; Goode et al. 1996b). Because both genes are required in the germ line, this leads to the clear prediction that they are involved in the production of functional Delta. Brainiac shows sequence similarity to Fringe, and related mammalian proteins have been characterized as glucosyltransferases (Yuan et al. 1997; Amado et al. 1999; Bruckner et al. 2000). This raises the interesting possibility that Brainiac is a glucosyltransferase that adds sugar residues to Delta, in much the same way that Fringe modifies Notch.

\section{Materials and methods}

Fly stocks used in this study

As a wild-type stock, we used $w^{1118}$ (Lindsley and Zimm 1992). Mutant chromosomes used in this study are $N^{55 e 11}$ FRT101/

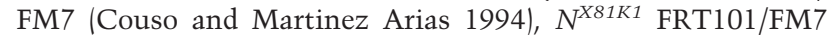
(Reddy et al. 1997), DI ${ }^{\text {M1 }}$ FRT82B/TM6B (de Celis et al. 1991), $S_{e r}{ }^{V \times 2} 2 D I^{\text {rev10 }}$ FRT82B/TM6B (Micchelli et al. 1997), Su(H)del47 FRT40A/CyO (Morel and Schweisguth 2000), $p s n^{C 1}$ FRT2A/ TM3 (Struhl and Greenwald 1999), E(spl) b32.2 FRT82B/TM6B (Schrons et al. 1992). $H^{25} /$ TM3 (Maier et al. 1992) was obtained from the Bloomington Stock Center and recombined onto an FRT82B chromosome using standard genetic techniques.

The $P$-lacW enhancer-trap lines that label different population of follicle cells $(1387 / 16,608 / 07,998 / 12,679 / 15)$ are insertions on the $3^{\text {rd }}$ chromosome from the Péter Deák collection (Deák et al. 1997; Deng, Bownes, González-Reyes and St. Johnston, unpubl.). A101 and PA77.1M3 (Bellen et al. 1989), were obtained from the Bloomington Stock Center and are LacZ insertions on the $3^{\text {rd }}$ chromosome.

Maternal germ line-specific expression of a GFP-Staufen fusion transgene was driven from the $\alpha 4$-tubulin promoter (Schuldt et al. 1998). The GFP-Staufen fusion protein localizes to the cytoplasm in nurse cells and at the posterior pole of the oocyte.

\section{Generation of clones}

To label homozygous clones in the follicle cells or germ line, we used the FRT-FLP technique in combination with GFP as a marker (the FRT and hsFLP stocks are described in Chou and Perrimon 1996). Flies expressing ubi-nlsGFP (Davis et al. 1995) show nuclear GFP expression in the follicle cells and in the germ line. FRT chromosomes marked with ubi-nlsGFP (FRT2A, FRT40A, and FRT82B) were kindly provided by Stefan Luschnig (Luschnig et al. 2000). GFP FRT101 was provided by Alfonso Martínez-Arias. Homozygous mutant clones in follicle cells were generated by heat shocking pupae or newly hatched female flies for $2 \mathrm{~h}$ at $37^{\circ} \mathrm{C}$, during 3 consecutive days. Adult females were placed on yeasted media at $25^{\circ} \mathrm{C}$ and dissected between 2 to 7 days after the last heat shock. Germ line clones were generated by heat shocking 3rd instar larvae and pupae using the same regime describe above.

All the 3rd chromosome enhancer trap lines were crossed to GFP FRT101, hsFLP22 flies. Male progeny of the genotype GFP FRT101, hsFLP22/+ P-lacZ/ + was then crossed to $N^{55 e 11}$ FRT101 females to generate females of the genotype $N^{55 e 1}$ FRT101/ GFP FRT101; hsFLP22/+ P-lacZ/+. Sometimes we used a hsFLP22 P[GFP-Staufen] to generate clones for the mutations of the right arm of the 3rd chromosome.

\section{Staining procedures}

Ovary dissections, and antibody and rhodamine-phalloidin stainings were performed as previously described (GonzálezReyes and St. Johnston 1998). OliGreen (Molecular Probes) staining was performed after the last wash for $10 \mathrm{~min}$ at $1 / 5000$ dilution in FasIII-stained egg chambers. Antibodies were used at the following concentrations: mouse monoclonal anti-FasIII 1/10 dilution (Patel et al. 1987); rabbit polyclonal anti-PhosphoHistoneH3 1/1000 (Upstate Biotechnology); rabbit anti- $\beta \mathrm{Gal}$ 1/2000 (Cappel); mouse monoclonal anti-N ${ }^{\mathrm{ICD}}$ (C179C6) 1/1000 (Fehon et al. 1991); mouse monoclonal anti-Dl (C594.9B) 1/100 (Diederich et al. 1994). Cy5- and Texas Red-conjugated secondary antibodies (Jackson) were used at $1 / 200$ dilution. All samples for confocal microscopy were mounted in Vectashield (Vector Laboratories). All confocal micrographs were collected using a BioRad MRC1024 scan head mounted on a Nikon E800 microscope. Adobe Photoshop and Micromedia FreeHand programs were used to process and assemble all figures.

\section{Acknowledgments}

We thank the following colleagues for the generous gifts of antibodies and fly stocks: Spyros Artavanis-Tsakonas, Sarah Bray, Julia Kaltschmidt, Thomas Klein, Stefan Luschnig, Alfonso Martínez-Arias, Nipam Patel, François Schweisguth, Gary Struhl, and the Bloomington Stock Center. We are grateful to A. Martínez-Arias, S. Bray, M. Furriols, S. Gregory, M.D., Martín Bermudo, I. Palacios, J. Pines, D. Santamaria and other members of the St. Johnston group for helpful insights during the course of this study. This work was supported by a HSFPO grant. D.StJ. is a Wellcome Trust Principal Research Fellow.

The publication costs of this article were defrayed in part by payment of page charges. This article must therefore be hereby marked "advertisement" in accordance with 18 USC section 1734 solely to indicate this fact.

\section{References}

Amado, M., Almeida, R., Schwientek, T., and Clausen, H. 1999. Identification and characterization of large galactosyltransferase gene families: Galactosyltransferases for all functions. Biochim. Biophys. Acta 1473: 35-53.

Artavanis-Tsakonas, S., Rand, M.D., and Lake, R.J. 1999. Notch signaling: Cell fate control and signal integration in development. Science 284: 770-776.

Bailey, A.M. and Posakony, J.W. 1995. Suppressor of Hairless directly activates transcription of Enhancer of split complex genes in response to Notch receptor activity. Genes \& Dev. 9: 2609-2622.

Bang, A.G., Bailey, A.M., and Posakony, J.W. 1995. Hairless promotes stable commitment to the sensory organ precursor 
cell fate by negatively regulating the activity of the Notch signaling pathway. Dev. Biol. 172: 479-494.

Baonza, A. and Garcia-Bellido, A. 2000. Notch signaling directly controls cell proliferation in the Drosophila wing disc. Proc. Natl. Acad. Sci. 97: 2609-2614.

Bellen, H.J., O'Kane, C.J., Wilson, C., Grossniklaus, U., Pearson, R.K., and Gehring, W.J. 1989. P-element-mediated enhancer detection: A versatile method to study development in Drosophila. Genes \& Dev. 3: 1288-1300.

Bender, L.B., Kooh, P.J., and Muskavitch, M.A. 1993. Complex function and expression of Delta during Drosophila oogenesis. Genetics 133: 967-978.

Berry, L.W., Westlund, B., and Schedl, T. 1997. Germ-line tumor formation caused by activation of $g l p-1$, a Caenorhabditis elegans member of the Notch family of receptors. Development 124: 925-936.

Brou, C., Logeat, F., Lecourtois, M., Vandekerckhove, J., Kourilsky, P., Schweisguth, F., and Israel, A. 1994. Inhibition of the DNA-binding activity of Drosophila Suppressor of Hairless and of its human homolog, KBF2/RBP-J $\kappa$, by direct proteinprotein interaction with Drosophila Hairless. Genes \& Dev. 8: 2491-2503.

Bruckner, K., Perez, L., Clausen, H., and Cohen, S. 2000. Glycosyltransferase activity of Fringe modulates Notch-Delta interactions. Nature 406: 411-415.

Calvi, B.R., Lilly, M.A., and Spradling, A.C. 1998. Cell cycle control of chorion gene amplification. Genes \& Dev. 12: 734-744.

Campos-Ortega, J.A. and Knust, E. 1990. Genetics of early neurogenesis in Drosophila melanogaster. Annu. Rev. Genet. 24: 387-407.

Chou, T.-B. and Perrimon, N. 1996. The autosomal FLP-DFS technique for generating germ line mosaics in Drosophila melanogaster. Genetics 144: 1673-1679.

Clark, I., Giniger, E., Ruohola-Baker, H., Jan, L., and Jan, Y. 1994. Transient posterior localisation of a kinesin fusion protein reflects anteroposterior polarity of the Drosophila oocyte. Cur. Biol. 4: 289-300.

Couso, J.P. and Martinez Arias, A. 1994. Notch is required for wingless signaling in the epidermis of Drosophila. Cell 79: 259-272.

Davis, I., Girdham, C.H., and O'Farrell, P.H. 1995. A nuclear GFP that marks nuclei in living Drosophila embryos: Maternal supply overcomes a delay in the appearance of zygotic fluorescence. Dev. Biol. 170: 726-729.

Deák, P., Omar, M.M., Saunders, R.D., Pal, M., Komonyi, O., Szidonya, J., Maroy, P., Zhang, Y., Ashburner, M., Benos, P., et al. 1997. P-element insertion alleles of essential genes on the third chromosome of Drosophila melanogaster: Correlation of physical and cytogenetic maps in chromosomal region 86E-87F. Genetics 147: 1697-1722.

de Celis, J.F., Mari-Beffa, M., and Garcia-Bellido, A. 1991. Cellautonomous role of Notch, an epidermal growth factor homologue, in sensory organ differentiation in Drosophila. Proc. Natl. Acad. Sci. 88: 632-636.

De Strooper, B., Annaert, W., Cupers, P., Saftig, P., Craessaerts, K., Mumm, J.S., Schroeter, E.H., Schrijvers, V., Wolfe, M.S., Ray, W.J., et al. 1999. A presenilin-1-dependent $\gamma$-secretaselike protease mediates release of Notch intracellular domain. Nature 398: 518-522.

Diederich, R.J., Matsuno, K., Hing, H., and Artavanis-Tsakonas, S. 1994. Cytosolic interaction between deltex and Notch ankyrin repeats implicates deltex in the Notch signaling pathway. Development 120: 473-481.

Fehon, R.G., Johansen, K., Rebay, I., and Artavanis-Tsakonas, S. 1991. Complex cellular and subcellular regulation of Notch expression during embryonic and imaginal development of Drosophila: Implications for Notch function. J. Cell Biol. 113: 657-669.

Forbes, A.J., Spradling, A.C., Ingham, P.W., and Lin, H. 1996. The role of segment polarity genes during early oogenesis in Drosophila. Development 122: 3283-3294.

Go, M.J., Eastman, D.S., and Artavanis-Tsakonas, S. 1998. Cell proliferation control by Notch signaling in Drosophila development. Development 125: 2031-2040.

Godt, D. and Tepass, U. 1998. Drosophila oocyte localisation is mediated by differential cadherin-based adhesion. Nature 395: 387-391.

González-Reyes, A., Elliott, H., and St. Johnston, D. 1995. Polarization of both major body axes in Drosophila by gurkentorpedo signalling. Nature 375: 654-658.

González-Reyes, A. and St. Johnston, D. 1994. Role of oocyte position in the establishment of anterior-posterior polarity in Drosophila. Science 266: 639-642.

. 1998a. Patterning of the follicle cell epithelium along the anterior-posterior axis during Drosophila oogenesis. Development 125: 2837-2846.

- 1998 b. The Drosophila AP axis is polarised by the cadherin-mediated positioning of the oocyte. Development 125: 3635-3644.

Goode, S., Wright, D., and Mahowald, A. 1992. The neurogenic locus brainiac cooperates with the Drosophila EGF receptor to establish the ovarian follicle and determine its dorsalventral polarity. Development 116: 177-192.

Goode, S., Melnick, M., Chou, T.-B., and Perrimon, N. 1996a. The neurogenic genes egghead and brainiac define a novel signaling pathway essential for epithelial morphogenesis during Drosophila oogenesis. Development 122: 3863-3879.

Goode, S., Morgan, M., Liang, Y.-P., and Mahowald, A.P. 1996b. brainiac encodes a novel, putative secreted protein that cooperates with Grk TGFa in the genesis of the follicular epithelium. Dev. Biol. 178: 35-50.

Greenwald, I. 1998. LIN-12/Notch signaling: Lessons from worms and flies. Genes \& Dev. 12: 1751-1762.

Jiang, Y.J., Aerne, B.L., Smithers, L., Haddon, C., Ish-Horowicz, D., and Lewis, J. 2000. Notch signalling and the synchronization of the somite segmentation clock. Nature 408: 475479.

Johnston, L.A. and Edgar, B.A. 1998. Wingless and Notch regulate cell-cycle arrest in the developing Drosophila wing. $\mathrm{Na}$ ture 394: $82-84$

Jordan, K.C., Clegg, N.J., Blasi, J.A., Morimoto, A.M., Sen, J., Stein, D., McNeill, H., Deng, W.M., Tworoger, M., and Ruohola-Baker, H. 2000. The homeobox gene mirror links EGF signalling to embryonic dorso- ventral axis formation through Notch activation. Nat. Genet. 24: 429-433.

Kimble, J. and Simpson, P. 1997. The LIN-12/Notch signaling pathway and its regulation. Annu. Rev. Cell Dev. Biol. 13: $333-361$.

Koch, E. and Spitzer, R. 1983. Multiple effects of colchicine on oogenesis in Drosophila: Induced sterility and switch of potential oocyte to nurse-cell developmental pathway. Cell Tissue Res. 228: 21-32.

Lane, M.E. and Kalderon, D. 1994. RNA localization along the anteroposterior axis of the Drosophila oocyte requires PKAmediated signal transduction to direct normal microtubule organization. Genes \& Dev. 8: 2986-2995.

Larkin, M.K., Holder, K., Yost, C., Giniger, E., and RuoholaBaker, H. 1996. Expression of constitutively active Notch arrests follicle cells at precursor stage during Drosophila oogenesis and disrupts the anterior-posterior axis of the oocyte. Development 122: 3639-3650. 
Larkin, M.K., Deng, W.-M., Holder, K., Tworoger, M., Clegg, N., and Ruohola-Baker, H. 1999. Role of Notch pathway in terminal follicle cell differentiation during Drosophila oogenesis. Dev. Genes Evol. 209: 301-311.

Lecourtois, M. and Schweisguth, F. 1995. The neurogenic Suppressor of Hairless DNA-binding protein mediates the transcriptional activation of the Enhancer of split complex genes triggered by Notch signaling. Genes \& Dev. 9: 2598-2608.

Lehmann, R., Jiménez, F., Dietrich, U., and Campos-Ortega, J.A. 1983. On the phenotype and development of mutants of early neurogenesis in Drosophila melanogaster. Roux's Arch. Dev. Biol. 192: 62-74.

Lindsley, D.L. and Zimm, G.G. 1992. The Genome of Drosophila melanogaster. Academic Press, San Diego, CA.

Luschnig, S., Krauss, J., Bohmann, K., Desjeux, I., and NussleinVolhard, C. 2000. The Drosophila SHC adaptor protein is required for signaling by a subset of receptor tyrosine kinases. Mol. Cell 5: 231-241.

Maier, D., Stumm, G., Kuhn, K., and Preiss, A. 1992. Hairless, a Drosophila gene involved in neural development, encodes a novel, serine rich protein. Mech. Dev. 38: 143-156.

Margolis, J. and Spradling, A. 1995. Identification and behavior of epithelial stem cells in the Drosophila ovary. Development 121: 3797-3807.

McClay, D.R., Peterson, R.E., Range, R.C., Winter-Vann, A.M., and Ferkowicz, M.J. 2000. A micromere induction signal is activated by $\beta$-catenin and acts through Notch to initiate specification of secondary mesenchyme cells in the sea urchin embryo. Development 127: 5113-5122.

Micchelli, C.A., Rulifson, E.J., and Blair, S.S. 1997. The function and regulation of cut expression on the wing margin of Drosophila: Notch, Wingless and a dominant negative role for Delta and Serrate. Development 124: 1485-1495.

Moloney, D.J., Panin, V.M., Johnston, S.H., Chen, J., Shao, L., Wilson, R., Wang, Y., Stanley, P., Irvine, K.D., Haltiwanger, R.S., et al. 2000. Fringe is a glycosyltransferase that modifies Notch. Nature 406: 369-375.

Morel, V. and Schweisguth, F. 2000. Repression by Suppressor of Hairless and activation by Notch are required to define a single row of single-minded expressing cells in the Drosophila embryo. Genes \& Dev. 14: 377-388.

Munro, S. and Freeman. 2000, M. The Notch signalling regulator Fringe acts in the Golgi apparatus and requires the glycosyltransferase signature motif DXD. Curr. Biol. 10: 813820.

Myster, D.L. and Duronio, R.J. 2000. To differentiate or not to differentiate? Curr. Biol. 10: R302-R304.

Neuman-Silberberg, F. and Schüpbach, T. 1993. The Drosophila dorsoventral patterning gene gurken produces a dorsally localized RNA and encodes a TGFa-like protein. Cell 75: 165174.

Oellers, N., Dehio, M., and Knust, E. 1994. bHLH proteins encoded by the Enhancer of split complex of Drosophila negatively interfere with transcriptional activation mediated by proneural genes. Mol. Gen. Genet. 244: 465-473.

Patel, N.H., Snow, P.M., and Goodman, C.S. 1987. Characterization and cloning of Fasciclin III: A glycoprotein expressed on a subset of neurons and axon pathways in Drosophila. Cell 48: 975-988.

Pokrywka, N. and Stephenson, E. 1995. Microtubules are a general component of mRNA localisation systems in Drosophila oocytes. Dev. Biol. 167: 363-370.

Reddy, G.V., Gupta, B., Ray, K., and Rodrigues, V. 1997. Development of the Drosophila olfactory sense organs utilizes cell-cell interactions as well as lineage. Development 124: 703-712.
Roth, S., Neuman-Silberberg, F.S., Barcelo, G., and Schüpbach, T. 1995. cornichon and the EGF receptor signaling process are necessary for both anterior-posterior and dorsal-ventral pattern formation in Drosophila. Cell 81: 967-978.

Ruohola, H., Bremer, K.A., Baker, D., Sedlow, J.R., Jan, L.Y., and Jan, Y.N. 1991. Role of neurogenic genes in establishment of follicle cell fate and oocyte polarity during oogenesis in Drosophila. Cell 66: 433-449.

Schrons, H., Knust, E., and Campos-Ortega, J.A. 1992. The Enhancer of split complex and adjacent genes in the $96 \mathrm{~F}$ region of Drosophila melanogaster are required for segregation of neural and epidermal progenitor cells. Genetics 132: 481503.

Schuldt, A.J., Adams, J.H.J., Davidson, C.M., Micklem, D.R., St. Johnston, D., and Brand, A. 1998. Miranda mediates the asymmetric protein and RNA localisation in the developing nervous system. Genes \& Dev. 12: 1847-1857.

Schweisguth, F. and Posakony, J.W. 1994. Antagonistic activities of Suppressor of Hairless and Hairless control alternative cell fates in the Drosophila adult epidermis. Development 120: $1433-1441$.

Schweisguth, F. and Lecourtois, M. 1998. The activity of Drosophila Hairless is required in pupae but not in embryos to inhibit Notch signal transduction. Dev. Genes Evol. 208: 19-27.

Sestan, N., Artavanis-Tsakonas, S., and Rakic, P. 1999. Contactdependent inhibition of cortical neurite growth mediated by Notch signaling. Science 286: 741-746.

Spradling, A. 1993. Developmental genetics of oogenesis. In The Development of Drosophila melanogaster (eds. M. Bate and A. Martinez-Arias), pp. 1-70. Cold Spring Harbor Laboratory Press, New York, NY.

Struhl, G. and Greenwald, I. 1999. Presenilin is required for activity and nuclear access of Notch in Drosophila. Nature 398: 522-525.

Theurkauf, W.E., Smiley, S., Wong, M.L., and Alberts, B.M. 1992. Reorganization of the cytoskeleton during Drosophila oogenesis: Implications for axis specification and intercellular transport. Development 115: 923-936.

Tworoger, M., Larkin, M.K., Bryant, Z., and Ruohola-Baker, H. 1999. Mosaic analysis in the Drosophila ovary reveals a common hedgehog-inducible precursor stage for stalk and polar cells. Genetics 151: 739-748.

Wei, Y., Yu, L., Bowen, J., Gorovsky, M.A., and Allis, C.D. 1999. Phosphorylation of histone $\mathrm{H} 3$ is required for proper chromosome condensation and segregation. Cell 97: 99-109.

$\mathrm{Xu}, \mathrm{T} .$, Caron, L.A., Fehon, R.G., and Artavanis-Tsakonas, S. 1992. The involvement of the Notch locus in Drosophila oogenesis. Development 115: 913-922.

Ye, Y., Lukinova, N., and Fortini, M.E. 1999. Neurogenic phenotypes and altered Notch processing in Drosophila presenilin mutants. Nature 398: 525-529.

Yuan, Y.P., Schultz, J., Mlodzik, M., and Bork, P. 1997. Secreted fringe-like signaling molecules may be glycosyltransferases. Cell 88: 9-11.

Zhang, Y. and Kalderon, D. 2000. Regulation of cell proliferation and patterning in Drosophila oogenesis by Hedgehog signaling. Development 127: 2165-2176.

Zhao, D., Clyde, D., and Bownes, M. 2000. Expression of fringe is down regulated by Gurken/Epidermal growth factor receptor signaling and is required for the morphogenesis of ovarian follicle cells. J. Cell Sci. 113: 3781-3794. 


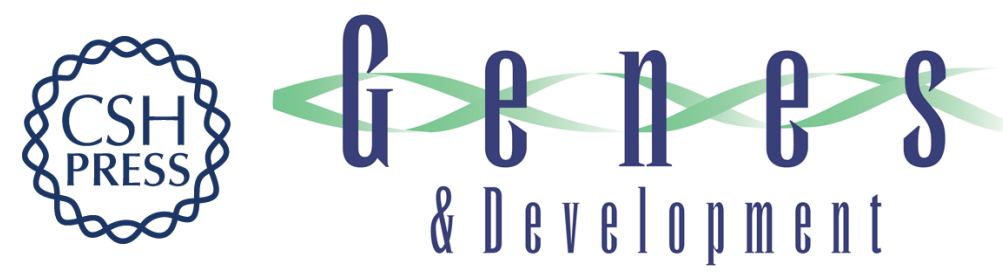

\section{Delta signaling from the germ line controls the proliferation and differentiation of the somatic follicle cells during Drosophila oogenesis}

Hernán López-Schier and Daniel St. Johnston

Genes Dev. 2001, 15:

Access the most recent version at doi:10.1101/gad.200901

\section{References This article cites 72 articles, 38 of which can be accessed free at: http://genesdev.cshlp.org/content/15/11/1393.full.html\#ref-list-1}

\section{License}

Email Alerting

Service

Receive free email alerts when new articles cite this article - sign up in the box at the top right corner of the article or click here.

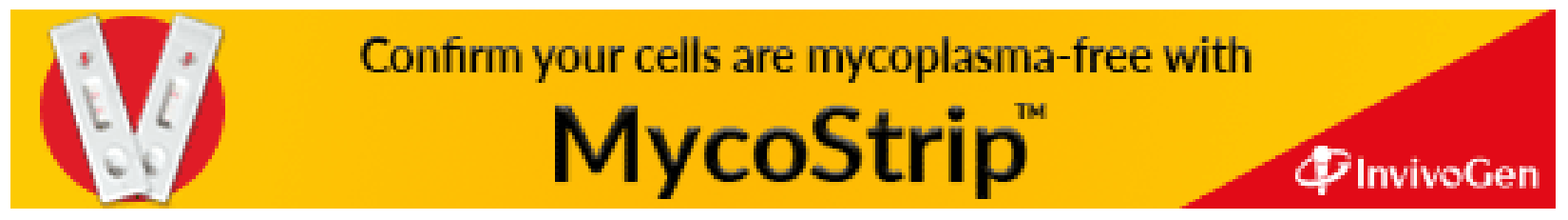

